\title{
Implicaciones de la teoría de la evolución en la filosofía*
}

Implications of Evolutionary Theory in Philosophy

\author{
Maximiliano Martinez ${ }^{\dagger}$
}

\begin{abstract}
Resumen
En este artículo expongo varias de las importantes implicaciones que la investigación en la evolución y sus procesos ha tenido en la reflexión filosófica. En la siguiente sección menciono, de manera general algunos de los aportes e influencias más relevantes en varias de las ramas de la filosofía, tales como teología, metafísica, epistemología, filosofía de la ciencia, filosofía de la mente y ética. Posteriormente, en las secciones 3, 4 y 5, analizo con mayor detalle algunas consecuencias particulares que ha tenido la investigación evolutiva en tres áreas fundamentales de la filosofia: ética, metafísica y epistemología (explicación científica). También mencionaré algunas críticas. En la sección final enumeraré algunas conclusiones.
\end{abstract}

Palabras clave: teoría de la evolución - filosofía - ética - metafísica - epistemología

\begin{abstract}
In this paper I analyze some of the important implications of evolutionary research on philosophical thinking. In the first section I discuss what I believe are the most relevant impacts of evolution in several areas of philosophy, such as theology, metaphysics, epistemology, philosophy of science, philosophy of mind and ethics. In sections 2, 3 and 4 I further analyze some of the specific consequences and influences that evolutionary thinking has had in ethics, metaphysics and epistemology and discuss some criticisms. The final section is devoted to some conclusions.
\end{abstract}

Keywords: theory of evolution - philosophy - ethics - metaphysics - epistemology

* Recibido: 30 de Abril de 2016. Aceptado con revisiones: 5 de Diciembre de 2016.

† Departamento de Humanidades, Universidad Autónoma Metropolitana-Cuajimalpa. Para contactar al autor, por favor, escribir a: mmartinez@correo.cua.uam.mx.

* Agradezco a Mario Casanueva, Alejandro Rosas y Maurizio Esposito por comentarios y sugerencias hechas a una versión previa. Metatheoria 8(1)(2017): 13-29. ISSN 1853-2322.

(C) Editorial de la Universidad Nacional de Tres de Febrero. Publicado en la República Argentina. 
It is a characteristic of the early twenty-first century that the philosophically most challenging science is no longer physics, but biology. And, of course, modern biology is essentially Darwinian.

Hösle e Illies (2005, p. 1)

\section{Introducción}

Si bien la idea de la transformación de las especies es anterior a Darwin y antes de El origen de las especies $^{1}$ (1859) existían varias reflexiones al respecto (Lamarck o Erasmus Darwin, por ejemplo), es con la publicación del Origen que la existencia de los procesos evolutivos empieza a ganar consenso entre los biólogos. Desde entonces, numerosos pensadores en diversas áreas del conocimiento han aplicado aspectos de la biología evolutiva (especialmente el proceso de selección) a sus investigaciones. Uno de estos campos es la filosofía.

En este texto expondré varias de las implicaciones que la investigación de la evolución y sus procesos han tenido en la reflexión filosófica. En la siguiente sección menciono, de manera general, algunos de los aportes e influencias más relevantes en varias de las ramas de la filosofía. Posteriormente, en las secciones 3, 4 y 5, analizaré con mayor detalle algunas consecuencias particulares que ha tenido la investigación evolutiva en tres áreas fundamentales de la filosofía: ética, metafísica y epistemología (explicación científica). También mencionaré algunas críticas. En la sección final enumeraré algunas conclusiones.

\section{Implicaciones de la biología evolutiva en la filosofía}

El impacto de la teoría evolutiva en la biología tuvo su punto natural de inicio con la publicación del Origen, y rápidamente se extendió a múltiples áreas diferentes de la biología, como la piscología, la sociología o la arqueología. ${ }^{2}$ Los tópicos del enfoque darwiniano sobre lo que hoy llamamos evolución de las especies son diversos, pero giran alrededor de dos ejes centrales: 1) la selección natural como el proceso evolutivo fundamental y, 2) la idea de origen compartido y ancestro común. Con el primero, Darwin propone una causa del diseño biológico y las adaptaciones a partir de un proceso natural mecánico y ciego, mientras que con el segundo explica la unidad de tipo (es decir, la semejanza morfológica entre especies) postulando una filiación compartida que se retrotrae a un ancestro común,

\footnotetext{
${ }^{1}$ La palabra 'evolución' aparece en el contexto del preformismo y significaba otra cosa; más bien se refería a un despliegue de las formas precontenidas en los gametos. En el Origen no aparece la palabra 'evolución', sólo existe el término 'evolucionado' que se presenta sólo una vez, en el famoso párrafo final: "There is grandeur in this view of life, with its several powers, having been originally breathed into a few forms or into one; and that, whilst this planet has gone cycling on according to the fixed law of gravity, from so simple a beginning endless forms most beautiful and most wonderful have been, and are being, evolved" (Darwin 1859, p. 490). Por otra parte, Beer sostiene: "During the eighteenth century, when the word [evolution] was used, it meant the stages through which a living being passes in the course of its development from egg to adult. That is, it gives an account of a single life span and remains within the pale of individual development. The term for this in biology is ontogeny. But evolutionary theory challenged the single life span as a sufficient model for understanding experience. In the 1830s the word evolution was used for the first time to describe the development of the species rather than of the individual. For this the biological term is phylogeny" (Beer 1983, p. 15).

2 Como afirma Ruse: "After Darwin published [the Origin], evolution as such became common sense". Sin embargo, continúa Ruse, el proceso de selección natural no gozó de la misma suerte: pocos lo adoptaron, siendo el lamarckismo o el saltacionismo procesos preferidos para explicar la evolución. Así mismo, surgieron dudas en torno al concepto de variación y de su estabilidad, dado que Darwin no tenía una teoría fuerte de la herencia. Otras críticas similares fueron las brechas en el registro fósil o la supuesta corta edad de la tierra, insuficiente para que actuara el lento proceso selectivo descrito por Darwin. Sólo hasta el re-descubrimiento de Mendel y el trabajo de biólogos como Fisher, Haldane y Wright en lo años 30, se abrió el espacio teórico y empírico para a) desvirtuar otros presuntos procesos evolutivos; b) ver a la selección natural en acción y, c) reivindicarla como el proceso evolutivo por antonomasia (Ruse 2009, pp. 6-9). Esta idea permaneció prácticamente inalterada durante la mayor parte del siglo XX.
} 
del cual se desprende la diversificación paulatina de las especies (Caponi 2011, Sober 2011). El reconocimiento del hecho de la evolución y estas dos propuestas (teóricas) resultaron revolucionaras, pues no sólo iban en contra de ideas atrincheradas y extendidas en los ámbitos científico y vernacular (como por ejemplo el argumento de la creación divina y la existencia de un diseñador omnipotente, la teoría de los múltiples orígenes de la vida o la idea del fijismo de las especies), sino que también proveían de una teoría bastante completa de la evolución.

La filosofía no fue ajena a este importante aporte científico y los postulados evolutivos darwinianos tuvieron una inmediata y cada vez mayor repercusión (la cual no ha cesado de ocurrir). Es así como encontramos, desde la misma época de Darwin y hasta nuestros días, una gran cantidad de pensadores que han adoptado entusiastamente enfoques evolutivos en sus investigaciones (y permitiendo la consolidación del proyecto naturalista en la filosofía), pero también encontramos no pocos críticos acérrimos que ven con enorme recelo la aplicación de los postulados de la evolución a cuestiones tradicionalmente teóricas.

En todo caso, en varias de las principales ramas de la filosofía encontramos comúnmente aproximaciones evolutivas que abordan importantes problemas. Tal es el caso de la ética, la epistemología o la filosofía de la ciencia. Estas no son las únicas áreas en donde el pensamiento evolutivo ha tenido importantes implicaciones, también lo ha hecho en la metafísica, en la filosofía de la mente o en la teología. Podría decirse, incluso, que una buena parte de la filosofía contemporánea es de la opinión de que no se puede hacer mucha reflexión teórica sustantiva si no se está al tanto de lo que dicen nuestras mejores teorías científicas. Así las cosas, para muchos, el enfoque evolucionista se convierte en una herramienta idónea para intentar resolver o replantear varios de los problemas filosóficos tradicionales. A continuación, se presentan, de manera general, algunas de las influencias de la teoría de la evolución en ramas centrales de la filosofía. ${ }^{3}$ La lista no pretende ser exhaustiva, pero sí reflejar varias de las implicaciones importantes de la teoría evolutiva en asuntos filosóficos de primer orden.

\subsection{Teología}

La prueba de la teología natural (del creacionismo o del diseño inteligente) para la existencia de Dios, basada en el argumento del diseño, pierde sentido al postularse a la selección natural como la causa del diseño de los seres vivos (Paley [1802] 1836, Bell 1834). Así, los patrones, mecanismos y procesos naturales que evidencian organización no requieren una intención detrás que los guíe (i.e., se puede llegar a un orden en la naturaleza sin un ordenador). ${ }^{4}$

\subsection{Metafísica}

Las implicaciones del pensamiento evolucionista en la metafísica son variadas. Menciono por ahora dos. La primera es el cuestionamiento que la teoría evolutiva hace a la metafísica analítica tradicional de las categorías usadas por ésta en sus análisis. Me refiero a conceptos tales como identidad, mereología, propiedad, substancia, relación, etc. Uno de los supuestos evolutivos es que nuestro aparato cognitivo, nuestra mente, evoluciona por selección natural como herramienta para ser usada en la supervivencia, permitiendo discriminar y clasificar el entorno de una manera particular y útil a este propósito (Pinker 1997, Mithen 1998). Ahora, si ésta es la función de nuestras capacidades cognitivas,

\footnotetext{
${ }^{3}$ Si bien Darwin mismo dudaba de sus propias capacidades filosóficas, escribió a lo largo de sus obras sobre una variedad de temas que siguen siendo foco de investigación filosófica actual: el origen de los sentimientos morales, el origen de la cooperación, el origen de la moral, la naturaleza de las emociones, el origen del conocimiento innato, la causa de las adaptaciones y la apariencia de diseño en las formas biológicas particulares (Lewens 2009). Como afirma Martinez-Contreras, "With Darwin we received the idea that every aspect of life, including instincts and minds, is subject to natural selection" (2011, p. 408).

${ }^{4}$ Guillaumin argumenta que Darwin seculariza la ciencia que, en la Inglaterra del XIX, se relacionaba con ideas teológicas, como la de Whewell (Guillaumin 2009, pp. 216-217).
} 
entonces nuestra mente no estaría diseñada para descubrir la estructura última de la naturaleza, el cual es el objetivo primario de la metafísica. En otras palabras, las categorías utilizadas por la metafísica analítica serían un reflejo, una mera extensión de las categorías fenoménicas o de sentido común que usa nuestra cognición para responder a presiones selectivas ancestrales, las cuales no serían adecuadas para desarrollar un análisis de la estructura fundamental del mundo. Por ejemplo, no hay una razón para pensar que la estructura sub-atómica de las partículas soporte las relaciones mereológicas que les atribuimos, esto es, no hay evidencia de que el mundo se divida en un dominio de objetos y propiedades (Ladyman \& Ross 2010).

Una segunda implicación de la teoría evolutiva en la metafísica, sostenida por varios filósofos y biólogos, es que con el pensamiento darwinista se refuta el esencialismo tradicional (platónico y aristotélico), al menos en lo que respecta al mundo viviente. Según varios autores (Mayr, Sober, Wuketits) Darwin muestra la inexistencia de tipos en biología y reemplaza la metafísica esencialista biológica por una de tipo poblacional. Sobre este punto volveré, de forma detallada, en la sección 5 .

\subsection{Epistemología}

La epistemología evolucionista es un intento por incorporar la perspectiva evolucionista en la teoría del conocimiento. Hay dos maneras usuales de hacerlo (Bradie 1986, 2012). La primera consiste en suponer un paralelo entre la evolución de nuestros rasgos fenotípicos y la evolución de nuestras capacidades cognitivas. Así, los humanos, al igual que las otras especies, hemos desarrollado capacidades cognitivas que son adaptativas. Aunque en este punto no hay mayor controversia, en donde sí hay discusión entre los epistemólogos evolucionistas es en torno al hecho de que si el sistema sensorial humano es un producto evolutivo, entonces este sea generalmente confiable. A este respecto, algunos teóricos afirman que este es de hecho el caso, pues si no tuviésemos creencias verdaderas hubiésemos sido eliminados por la selección natural (Lorenz 1977). Por el contrario, otros arguyen que no necesariamente: las creencias adaptativamente ventajosas pueden ser falsas (Ruse 1986).

Otra forma de epistemología evolucionista es la propuesta por Popper (1972), Toulmin (1972), Campbell (1974) y Hull (1988). Esta explica el desarrollo del conocimiento científico en analogía con el proceso de evolución orgánica en la medida en que ambos están sometidos a algún tipo de selección natural. El conocimiento emerge a través de un proceso que involucra variación, selección y retención. La primera, cuando varias hipótesis son conjeturadas. La segunda, cuando éstas son comprobadas o refutadas. La tercera, cuando se retiene a aquella o aquellas que pasaron la comprobación. Esta retención, que se hace en la memoria o en documentos, es conocimiento, el cual es base de posterior conocimiento e investigación. ${ }^{5}$

Por otro lado, la investigación cada vez más profunda en la evolución y la evidencia empírica creciente de sus procesos trae importantes consecuencias para la noción de explicación científica. El reduccionismo es cuestionado seriamente y la explicación basada exclusivamente en las causas eficientes mecanicistas empieza a lucir parcial y en muchos casos insuficiente para capturar la complejidad de lo viviente. Sobre este punto volveré con más detalle en la sección 4.

\subsection{Filosofía de la ciencia como área}

Con la consolidación de la teoría evolutiva, bajo la bandera de la así llamada 'teoría sintética', principalmente desde la década de los 30 y los 40, el foco casi exclusivo de investigación en la filosofía de la ciencia deja de ser la física y la biología empieza paulatinamente a tomar buena parte de su lugar. La década de los 70 fue crucial para que la filosofía de la biología se convirtiera en una disciplina

\footnotetext{
5 Para una crítica a este tipo de epistemología evolucionista, véase Ruse (1986). Este último considera que la analogía propuesta no se
} sostiene, convirtiéndose el ejercicio en una simple metáfora. 
independiente: varios filósofos de primer orden se dedican a la reflexión de los procesos evolutivos y las prácticas en biología, al tiempo que numerosos biólogos se concentran en reflexiones teóricas. Aparecen textos introductorios a la filosofía de la biología, así como compilaciones. Esta tendencia continúa en la década de los 80 , sumándose otro buen número de teóricos, creándose tanto la primera revista especializada en filosofía de la biología (Biology EF Philosophy), como la primera asociación internacional del tema (ISHPSSB). Desde entonces, se abren numerosas posiciones para filósofos de la biología en muchos departamentos de filosofía a lo largo del orbe, incluyendo a los más prestigiosos. La producción de textos en el tema ha sido enorme. Hoy en día, la filosofía de la biología es una de las áreas de investigación de mayor auge en la filosofía de la ciencia (véase Hull 2008, Abrantes 2011).

\subsection{Filosofía de la mente}

Las explicaciones evolucionistas se aplican generalmente cuando se trata de preguntas sobre la naturaleza fisiológica de los organismos, pero el mismo Darwin, tanto en El origen del hombre (1871) como en La expresión de las emociones en los animales y el hombre (1872), aplicó su teoría a los fenómenos psicológicos y conductuales. Esta idea fue retomada por varios autores desde entonces (James, Piaget, Hamilton). Con ese espíritu, en 1975 Edward O. Wilson publicó su libro titulado Sociobiology: The New Sintesis (1975), donde sienta las bases de la sociobiología, la cual trata de dar cuenta de la conducta animal (incluyendo a los humanos) a partir de la selección natural. Debido a múltiples críticas dicho proyecto se viene abajo, dando lugar a la psicología evolucionista, área de investigación producto del trabajo interdisciplinar entre la teoría evolucionista y la psicología cognitiva ${ }^{6}$ (y otras disciplinas, como la antropología, la arqueología o la primatología). Otro insumo importante para el desarrollo de la psicología evolucionista fue la teoría de la modularidad mental propuesta por Fodor (1983), según la cual buena parte de la mente (la percepción y el lenguaje) consiste en una serie de programas que llamó módulos y que tienen cada uno un propósito particular y sus propias reglas. Posteriormente Tooby y Cosmides $(1992,2005)$ retomaron la idea de una mente modular y argumentaron a favor de la existencia de una enorme cantidad de módulos mentales. Esto se conoce como la tesis de la modularidad masiva de la mente. Al aunar estas ideas con la biología evolucionista, tenemos la tesis de que los distintos y múltiples módulos mentales son, cada uno, adaptaciones diseñadas por la selección natural para resolver algún problema adaptativo. Algunos de estos módulos evolucionaron desde el ancestro común entre los reptiles y los humanos, mientras que otros son más recientes y exclusivamente humanos. Ello implicaría, que buena parte de nuestras capacidades mentales son adaptaciones.

\section{6. Ética}

¿Podemos explicar los comportamientos morales humanos y su psicología subyacente a partir de la teoría de la evolución por selección natural? Este es un proyecto tanto importante como controversial. Una buena parte de los biólogos evolucionistas dedicados al tema lo ven como un proyecto viable en muchos sentidos, principalmente porque consideran que la cooperación es una realidad biológica muy extendida, que tiene una explicación en términos adaptativos. Entre filósofos el proyecto es más polémico pero su aceptación es creciente, haciendo que el enfoque de ver a la moral como una adaptación sea aplicada en varios de los temas centrales de la ética. Abordo estos temas en la siguiente sección.

En las tres siguientes secciones retomo y abundo en varios de los temas mencionados hasta ahora. En la siguiente sección me concentro en algunas de las implicaciones de la teoría evolutiva en la ética.

\footnotetext{
${ }^{6}$ Esta última concibe a la mente, grosso modo, como una especie de programa de computadora cuya función es procesar información.
} 
Posteriormente, en las secciones 4 y 5, hago lo propio con los temas de explicación científica y metafísica, respectivamente. Dejo la sección final para señalar algunas conclusiones generales.

\section{Implicaciones de la teoría evolutiva en la reflexión ética}

La influencia y aplicación de la teoría evolutiva ha sido amplia en las investigaciones en filosofía moral, sobre todo a partir de la concepción de que nuestro sentido ético podría ser un producto de la selección natural, dándole así un carácter adaptativo a la moralidad. Esta idea permite abordar, desde una perspectiva evolutiva, temas centrales de la ética como la distinción entre justificación y explicación moral, el asunto de la motivación o el debate metaético entre cognitivistas y no cognitivistas o entre realismo y escepticismo. Así mismo, al resolverse la paradoja del altruismo es posible sentar las bases para una explicación natural del origen de la moralidad. El mismo carácter normativo de la moral tampoco es ajeno a la influencia de los estudios evolutivos. Es así como existe hoy en día un importante programa de investigación conocido como naturalismo ético. A continuación me concentro en estos puntos.

\subsection{Altruismo y cooperación}

El altruismo y la cooperación son dos conceptos fundamentales para la ética, toda vez que reflejan dos comportamientos que, me atrevería a decir, son fomentados y prescritos por cualquier código moral de cualquier sociedad o grupo a lo largo de la historia humana. Esto los convierte no sólo en componentes básicos de la moral, también los ubica en primer plano como objeto de estudio tanto metaético como normativo. Darwin mismo se percató de esto, razón por la cual ofrece una explicación de la evolución de la cooperación y altruismo humanos en El origen del hombre (1871), a partir del proceso de selección natural. ${ }^{7}$ Desde entonces, no han cesado las explicaciones del mismo tenor, dando paso a la línea de investigación conocida como ética naturalista evolucionista. ¿Qué tiene que decir la investigación en biología evolutiva y selección natural al respecto del altruismo y la cooperación? De acuerdo con el proyecto naturalista, mucho: el origen, evolución y permanencia de tales rasgos conductuales debe explicarse a partir de nuestras mejores teorías científicas, en este caso, la teoría de la evolución por selección natural. Así, la investigación en filosofía moral (al menos en buena parte del campo metaético) se ve fuertemente influida por el pensamiento evolucionista. Esto, aunado a la evidencia de comportamientos cooperativos en otras especies, refuerza la idea de que la cooperación y el altruismo son productos naturales y que para comprenderlos y comprender su papel en la moral es necesario conocer su evolución.

Ahora bien, el mismo Darwin se percató de una aparente tensión entre el proceso de selección natural y la existencia del altruismo, lo que ha llevado a muchos a señalar la existencia de una paradoja (la cual marcó buena parte de la investigación biológica de la cooperación desde los 50). ¿En qué consiste la paradoja del altruismo? Un comportamiento altruista es aquel que beneficia a otro organismo y que va en detrimento del propio organismo que lo lleva a cabo. Ahora, si la selección natural tiende a eliminar a aquellos organismos que tienen un menor desempeño en la supervivencia y la reproducción, podría predecirse la desaparición de los altruistas ${ }^{8}$ ¿Cómo se explica entonces que haya organismos que se comportan de manera altruista, entre ellos los humanos? Darwin ofreció una solución a este asunto apelando a lo que se conoce como selección de grupos: para él, una tribu con

${ }^{7}$ En el capítulo cuatro de El origen del hombre (1871) Darwin expone de manera sistemática cómo los sentimientos morales pudieron haber evolucionado en los humanos a partir de capacidades cognitivas y emociones que poseyeron nuestros ancestros (una reflexión claramente metaética).

${ }^{8}$ En un escenario de franca lucha por la existencia, como propone Darwin para el mundo de lo viviente, un organismo que otorgue recursos propios a otro sin recibir nada a cambio, se encuentra en clara desventaja y propende por su propia extinción. 
integrantes más fieles, cooperativos y con un alto grado de sacrificio, tendrá éxito sobre otra tribu cuyos integrantes carezcan de tales virtudes. Es decir, en la competencia a nivel de grupos, aquellos que posean individuos cooperativos y altruistas tendrán un mayor éxito que los grupos que no los posean, garantizando la supervivencia de los individuos altruistas y la permanencia de sus comportamientos a través de la evolución. ${ }^{9}$ Aunque esta explicación gozó de gran simpatía por mucho tiempo en la biología evolutiva (el trabajo de W. Edwards de 1962 es un clásico al respecto; véase Borrello 2012), desde la década de los sesenta empezó a perder vigencia en contraposición a explicaciones que apelaban a la selección individual. ${ }^{10}$ En un influyente artículo, Axelrod y Hamilton (1981) mostraron, a partir de la teoría de juegos y el dilema del prisionero, que la cooperación puede evolucionar entre individuos no emparentados porque cooperar paga. En otras palabras (y como ya habían señalado Hobbes y Hume) la ayuda mutua trae beneficios a los cooperantes que le son ajenos a los individuos no cooperantes (por más prósperos que éstos sean). Así, la cooperación sería una estrategia adaptativa individual exitosa. Para que evolucione esta debe ser, claro está, discriminada: se debe cooperar sólo con individuos que cooperen a su vez.

Robert Trivers (1971), en otro artículo fundamental en este tipo de investigaciones, intenta mostrar cómo el altruismo evoluciona, haciendo una salvedad: el altruismo que puede evolucionar es el recíproco, nunca el indiscriminado. Hay dos asuntos muy importantes del trabajo de Trivers. Por un lado muestra que el altruismo, si es recíproco, traerá beneficios posteriores a los individuos que ejecutan actos altruistas de manera consistente y no indiscriminada. Por otro lado, Trivers especula de manera interesante cómo habrían evolucionado los sentimientos morales humanos, llegando incluso hasta aquellos que consideramos propios de la moralidad, como el sentido del deber, la indignación, el deseo de ayudar a otros, la lealtad, etc. Los trabajos de Axelrod y Hamilton (1981), y Trivers (1971), suponen un punto de partida importante para el proyecto naturalista de la moral, al ofrecer una teoría del origen y evolución de la cooperación, el altruismo y los sentimientos morales. Sin embargo, los trabajos de Sober y Wilson (1998) y Wilson y Wilson (2008), entre otros, han reintroducido con fuerza la selección de grupos de nuevo en la discusión del altruismo (véase Birch \& Okasha 2015).

\subsection{El juicio moral y la metaética}

Cuando los filósofos nos preguntamos sobre la fuente de la moralidad, nos podemos estar refiriendo a dos cosas distintas: 1) la pregunta por el origen de la moral y 2) la pregunta por la fundamentación de nuestras prácticas morales. La primera es de naturaleza descriptiva y exige una respuesta con forma de explicación; la segunda, en cambio es de naturaleza normativa y exige una respuesta con forma de justificación (por qué consideramos que una acción es correcta en vez de otra?). A pesar de que toda justificación tiene en última instancia la forma de una explicación, no toda explicación es una justificación (Rosas 2000), lo cual es especialmente cierto en el terreno de la moral. El dar una explicación sobre el origen de un patrón de conducta o sobre una norma moral no es lo mismo que justificarla. Por ejemplo, cuando explicamos las motivaciones psicológicas de un asesino, no lo estamos justificando. A partir de las teorías evolutivas se han hecho grandes contribuciones para responder a la pregunta descriptiva, pero a la vez se ha intentado responder a la pregunta normativa que se desprende desde este tipo de teorías. Por ejemplo, algunos filósofos (Joyce 2006, Kitcher 2006) han explorado la posibilidad de que nuestra capacidad de hacer juicios morales sea producto del proceso evolutivo al conferir ventajas adaptativas en nuestros ancestros. De ser así, dicha capacidad no sería solamente un

\footnotetext{
9 La interpretación estándar es que Darwin apeló a la selección de grupos. Sin embargo, encontramos autores que leen los pasajes de Darwin de manera diferente, argumentando que Darwin en realidad no defendió la selección de grupos en este punto, sino la individual (véase Ruse 1980, Gould 2002, Rosas 2004).

${ }^{10}$ Principalmente desde la publicación del influyente libro Adaptation and Natural Selection (Williams 1966), en donde se hace una fuerte defensa de la selección individual.
} 
producto cultural, sino parte de la naturaleza humana, lo cual tendría interesantes implicaciones filosóficas. Se ha llegado más lejos aún, al preguntarse si habría adaptaciones emocionales que influyan en el contenido de nuestros juicios morales (Greene 2006, 2015).

Por otro lado, dentro del debate entre los cognitivistas y no cognitivistas en la psicología moral, la teoría evolutiva también ha tenido implicaciones interesantes. A partir de modelos evolutivos aplicados y de trabajo empírico (i.e. desde encuestas hasta escaneos cerebrales), algunos investigadores han llegado a la conclusión de que la manera en que las personas hacen juicios morales (o al menos una buena mayoría) no es autónoma ni es hecha a través de un razonamiento deductivo, sino que es resultado una 'racionalización post-hoc' (Haidt 2001). Lo que pretenden mostrar estos estudios es que al estar ante un dilema moral las personas emiten un juicio ético inmediatamente, como producto de una emoción o un 'instinto moral (producto a su vez de la historia evolutiva) y después tratan de ofrecer una justificación de su juicio. De ser cierta esta tesis, bastante controversial para la ética tradicional, nuestras acciones morales no provendrían de una reflexión moral juiciosa y previa (que es como entendemos tradicionalmente a la acción moral y su razón, precedida de una reflexión), sería más bien un reflejo automático implantado por la selección natural. ${ }^{11}$

El que el contenido de nuestros juicios morales sea un producto evolutivo tiene implicaciones para la metaética. Sharon Street (2006) argumenta que el darwinismo enfrenta a los realistas morales con un serio dilema: caer en un escepticismo moral o rechazar el realismo moral (en pos de un constructivismo). Las investigaciones y estudios de los autores mencionados implican que nuestro sentido moral está saturado con la influencia de nuestra historia evolutiva para que actuemos de manera que optimicemos nuestra aptitud biológica (fitness), no para descubrir verdades morales (esto en claro paralelo con la crítica a la metafísica tradicional mencionada arriba, en 2.2). Esto nos lleva a desconfiar de nuestras intuiciones morales como objetivas, acarreando un escepticismo moral. Por lo que si queremos evitarlo, dice Street, debemos optar por un constructivismo anti-realista.

\subsection{Algunas críticas}

Una de las críticas más influyentes que se han hecho al proyecto naturalista de la moral la formuló el filósofo G.E. Moore en 1903 (Moore [1903] 1997). Moore sostuvo que es equivocado intentar identificar o definir las propiedades morales, como la bondad, en términos naturalistas (o en cualquier otro tipo de términos), pues ellas son indefinibles, objetivas y no naturales. Moore proyectó sus críticas desde dos flancos. Uno: el famoso argumento de la pregunta abierta, en donde reduce al absurdo cualquier intento de definición de bueno. Dos: sostuvo un intuicionismo ético que postulaba la objetividad de las propiedades morales, no naturales pero cognoscibles a partir de nuestro particular sentido moral.

Otra crítica, no menos importante y clásica, proviene de un famoso pasaje del Tratado de Hume, en donde aparentemente se señala la imposibilidad lógica de pasar de premisas descriptivas a premisas normativas (del es al debe) lo que, para muchos intérpretes, demuestra la autonomía de la ética con respecto a la ciencia. ${ }^{12}$

Una crítica más reciente proviene del rechazo inicial que provocó el proyecto sociobiológico propuesto por Wilson $(1975,1978)$. Wilson afirmó, literalmente, que había llegado la hora de 'quitarle la investigación de la ética a los filósofos y entregársela a los biólogos'. Wilson argumentaba que la mayoría de los comportamientos humanos (aversión al incesto, promiscuidad, hostilidad hacia los extraños) eran adaptaciones genéticas (abarcando los campos metaético y normativo de la moral). La crítica no se hizo esperar, tanto por parte de filósofos como de biólogos. Por un lado, se señalaba el

\footnotetext{
${ }^{11}$ Vale la pena mencionar que para Haidt no todas las intuiciones morales son producto de la selección natural, también las hay de tipo cultural.

12 Para una respuesta a Moore y a Hume, en defensa del naturalismo moral, véase Martínez (2003).
} 
nocivo determinismo genético presente en la sociobiología (que de ser cierto, haría prácticamente imposible cambiar nuestros comportamientos naturales, justificando el status quo social). Dos, se señaló también el evidente "panglossianismo" presente en la mayoría de las explicaciones sociobiológicas, en donde sin mayor evidencia empírica se recreaba una historia adaptativa para cualquier comportamiento humano. A partir de este tipo de críticas, la sociobiología cayó en desgracia, haciendo que los posteriores intentos de aplicar la teoría evolutiva al campo de la moral se refinaran, evitando el adaptacionismo extremo, buscando mayor evidencia empírica, y concentrándose mayormente (aunque no exclusivamente) en el campo metaético. ${ }^{13}$

\section{Implicaciones de la teoría evolutiva en la explicación científica}

La comprensión de cómo opera el proceso de selección natural en la evolución tuvo importantes implicaciones en la forma de entender la causalidad natural-mecanicista y modificó en diversos aspectos el concepto de explicación científica. Veamos algunos de los puntos centrales de esta modificación.

\subsection{Explicaciones teleológicas y causas últimas}

Teleology is a mode of explanation in which the presence, occurrence, or nature of some phenomenon is explained by appeal to the goal or end to which it contributes.

Walsh (2008, p. 113)

Antes de Darwin la causa usualmente esgrimida (si bien no la única) para explicar el diseño biológico, es decir, las adaptaciones y rasgos funcionales de los organismos, era la propuesta por la teología natural: el diseño debe tener como causa, necesariamente, un diseñador (Paley [1802] 1836). Esta hipótesis, en un caso de inferencia a la mejor explicación, resultaba a todas luces la más plausible, comparada a la del diseño por azar: los organismos y sus complejos diseños funcionales se generaron aleatoriamente en la naturaleza. Dada la complejidad y especialización de las adaptaciones, resultaba altamente implausible que no hubiese una mente omnipotente detrás, capaz de concebir estructuras especializadas para que cumplieran exitosamente un fin.

Teniendo en cuenta esto, no sorprende el impacto que tiene la teoría darwiniana al proponer a la selección natural como la causa del diseño biológico, sentando las bases de una cosmovisión estrictamente naturalista. Darwin muestra cómo la naturaleza ciega puede, a través de un proceso permanente y cíclico de variación, selección y herencia, generar adaptaciones altamente especializadas que cumplen una función, erradicando así el recurso a un diseñador consciente. Desde la década de los 30 y hasta nuestros días, la opinión mayoritaria es que la selección natural es un proceso poderoso, capaz de conformar la forma especializada y funcional de los seres vivos. ${ }^{14}$ Nótese en todo caso que la selección natural no deja de ser un proceso enteramente natural, mecánico y ciego. De esta forma se da soporte al creciente proyecto naturalista en todos sus sentidos (ontológico, epistemológico y metodológico). ${ }^{15}$ En pocas palabras: la teoría de la evolución naturaliza la teleología. O, como afirman

${ }^{13}$ Postulando principios y reglas de comportamiento abstractas innatas, más que comportamientos particulares. Afirma Hauser: "We are born with abstract rules or principles, with nurture entering the picture to set the parameters and guide us toward the acquisition of particular moral systems" (2006, p. 165).

${ }^{14}$ Sin embargo, críticas recientes provenientes desde la Evo-Devo y la teoría de sistemas atacan fuertemente el papel causal positivo de la selección natural. En Martínez y Moya (2011) respondemos las críticas y argumentamos que la selección natural es un proceso causal multinivel generador de complejidad.

${ }^{15}$ Como afirma Walsh (2008, p. 113), la explicación teleológica fue suprimida de las ciencias fundamentales, como la física, haciendo que el modelo de explicación dominante fuera el mecanicista. Por este motivo, desde gran parte de la filosofía de la ciencia, guiada por el 
Barahona y Martínez (1998), retomando lo expuesto por Asa Gray en 1874, "la gran aportación del darwinismo a la ciencia consiste en mostrarnos la manera en que la teleología puede reincorporarse como un recurso explicativo en la ciencia” (1998, p. 422). Es así como, a partir de la comprensión paulatina de la operación del proceso de selección natural, el concepto de explicación científica se reformula y expande, para incluir a la teleología natural (es decir, la definición de explicación científica incluye ahora a las explicaciones de tipo teleológico natural sustentadas en la selección natural). El que la naturalización de la teleología permita comprender el diseño biológico y explicarlo científicamente es un cambio fundamental en la epistemología y la filosofía de la ciencia.

Veamos ahora otro punto, directamente relacionado con el anterior. Una vez comprendido que la teleología natural, ocasionada por los procesos selectivos, tiene un papel causal fundamental en la conformación particular de los seres vivos, el concepto de causalidad científica también se expande. ¿De qué modo? La ciencia acepta ahora tanto explicaciones en términos de causas mecanicistas como explicaciones en términos de causas remotas. De esta forma, las explicaciones funcionales, aparentemente paradójicas para la naturaleza (en donde el efecto precede a la causa), cobran pleno sentido científico, pues la selección natural es capaz de explicar la aparente finalidad de lo viviente. Esto se ve reflejado en la distinción entre causas próximas y últimas que, con inspiración aristotélica, propone Mayr en su influyente texto de 1961 "Cause and Effect in Biology" ${ }^{16}$ Veamos sus puntos principales. Mayr señaló la existencia de una separación entre dos áreas de la biología, la biología funcional y la biología evolutiva. Para Mayr, aunque tienen varios puntos de contacto, estas dos disciplinas son tan diferentes que cada una tiene sus propios métodos, objetivos y conceptos básicos (Mayr 1961). La biología funcional tiene que ver con la operación e interacción de elementos estructurales: moléculas hacia órganos y organismos. La pregunta permanente aquí es ‘cómo?' (How?) y la aproximación científica del biólogo funcional es "esencialmente la misma del físico o del químico" (Mayr 1961, p. 1502). Por el contrario, la cuestión básica en la biología evolutiva es 'ipor qué?' (Why?), es decir: "encontrar las causas [históricas] de los rasgos existentes y de las adaptaciones particulares es la principal preocupación del biólogo evolutivo” (Mayr 1961, p. 1502). Para Mayr, este último estudia el porqué de la biodiversidad, así como el porqué de la evolución de las adaptaciones. El punto central aquí es que, para Mayr, esta distinción se desprende una diferencia de las causas estudiadas por cada disciplina: a la biología funcional le conciernen las causas próximas (causas fisiológicas, inmediatas), a la biología evolutiva las causas últimas (causas selectivas, históricas): "es evidente que el biólogo funcional analizará las causas próximas, mientras que el biólogo evolutivo analizará las causas últimas” (Mayr 1961, p. 1503). Así, es posible explicar científicamente los hechos de la naturaleza a partir tanto de causas próximas (físico-químicas) como de causas últimas (evolutivas). De esta forma, vemos cómo el concepto de causalidad usado en la investigación científica se expande: es posible explicar en términos de causas próximas, pero también en términos de causas últimas e históricas. ${ }^{17}$

reduccionismo y la hipótesis de la unidad de la ciencia (Oppenheim \& Putnam 1958), se sostuvo que un modo de explicación señalada como ilegítima en la física no podría usarse en ninguna ciencia (véase también Martínez 1998). Una idea similar se encontraba ya en Kant. En la Crítica del juicio, Kant afirmaba que la teleología organizada que adjudicamos a los organismos no podía ser un principio objetivo de la naturaleza, sino uno subjetivo de la razón. Por otro lado, varios autores han defendido que Kant ya proponía, desde una perspectiva sistémica, un tipo de teleología natural que no necesitaba de diseñadores. Para una buena discusión del enfoque kantiano, véase Ginsborg (2006). Por su parte, Nagel defendía la posibilidad de dar explicaciones fisicoquímicas exhaustivas del mundo viviente. Nos dice en su influyente artículo de 1951: "The central thesis of this paper is that none of the argument advanced by organismic biologists establish the inherent impossibility of physico-chemical explanations of vital processes" (1951, p. 337). No sobra recordar que Nagel defendía que una explicación se consideraba científica solo si seguía el modelo nomológico-deductivo (Nagel 1961). Hoy día hay fuertes defensores del reduccionismo, como Rosenberg (véase por ejemplo su libro de 2006). Para un examen detallado de la noción de función y de las explicaciones funcionales, véase Caponi (2012), Diéguez (2012).

${ }^{16}$ Recordemos que Aristóteles se propuso explicar la naturaleza a partir de cuatro factores causales: causa material, causa formal, causa eficiente y causa final. Estas dos últimas son las que corresponderían a la distinción usada por Mayr.

${ }^{17}$ Otra implicación que para la explicación científica tiene el estudio de los procesos evolutivos es el abandono de la idea determinista de causalidad. En su influyente libro de 1912, Loeb defiende que el patrón de explicación científica por antonomasia, para lo biológico, es 


\subsection{Algunas críticas}

Varios autores como Mayr (2002) o Hull (1969) defienden que desde la teoría evolutiva no se naturaliza a la teleología, más bien ella se erradica de la biología. Para Mayr la naturaleza no es teleológica sino teleonómica. Según este autor, la palabra 'teleonomía' no refiere de ninguna manera a una 'intención' subyacente, como si lo hace la palabra 'teleología'. La palabra, 'teleología naturalizada' sería, desde esta postura, un oxímoron. Un proceso teleológico siempre presupone una mente que piensa en un fin, por lo que la evolución por selección natural no puede admitirla. Por el contrario, según Mayr, "teleonomía” refiere a un proceso de generación equiparable al teleológico, pero sin ningún objetivo predeterminado, es decir, sin direccionalidad ni finalidad alguna. Sin embargo, a mi modo de ver esta discusión es más terminológica que otra cosa. Encuentro más apropiado hablar de teleología naturalizada: este concepto captura las características mecánicas del proceso señaladas por Mayr, mientras que señala a su vez la ausencia de cualquier intencionalidad subyacente y, finalmente, enfatiza uno de los grandes logros de la teoría evolutiva para el conocimiento. Asimismo, concuerdo con Walsh (2008) en que las explicaciones teleológicas son indispensables en biología.

Con respecto a la distinción de causas próximas y últimas, en otros lugares he argumentado (Martínez \& Esposito 2014) que, de acuerdo a la investigación actual en varios campos de la biología, la dicotomía parece no tener una correspondencia ontológica garantizada en todos los casos. La clasificación que para Mayr fue en principio una herramienta heurística útil (útil para señalar dos tipos de prácticas científicas y dos tipos de explicación), terminó por reificarse y adquirir un exagerado estatus ontológico. El que las causas en la naturaleza se dividan tajantemente en dos clases no relacionadas, como parece defender Mayr (1994), no concuerda con la investigación más reciente en biología (por ejemplo desde la biología de sistemas, la construcción de nicho o la morfología genérica; véase también Laland et al. 2011).

\section{Implicaciones de la teoría evolutiva en la metafísica}

Como mencioné arriba en la sección dos, las implicaciones que la teoría evolutiva ha tenido en la metafísica son de un amplio espectro. Por un lado, se cuestiona la manera tradicional de hacer metafísica y sus categorías, así como el análisis de conceptos e intuiciones: no hay garantía de correspondencia de aquellos con la estructura última del mundo. Por otro lado, según varios autores, el logro más importante de la teoría de la evolución es refutar el esencialismo y develar la verdadera ontología del mundo viviente. Veamos en qué consiste esta posición.

\subsection{Pensamiento tipológico y poblacional: el reto al esencialismo}

Diversos autores de la filosofía de la biología y la teoría evolutiva, principalmente Ernst Mayr y Elliott Sober, han señalado la existencia de una dicotomía entre el pensamiento poblacional y el pensamiento tipológico (Mayr, uno de los arquitectos de la nueva síntesis y una de las figuras más prominentes de la teoría evolutiva, introdujo el término 'pensamiento poblacional', en los cincuentas, para referirse al aporte original que hacía Darwin a la filosofía). Mayr y Sober sostienen que Darwin postuló un nuevo marco de análisis y de investigación para entender la realidad natural, al introducir el enfoque poblacional en biología. Según ellos, este tipo de aproximación científica está soportado en una visión

el mecanicista (i.e. fisico-químico). Idea que es retomada y reformulada por Nagel (véase Martínez 1998). Mucho del reduccionismo del siglo XX refleja este espíritu (un ejemplo es Rosenberg 2006). Como afirma Martínez: "Una de las ideas más persistentes en el fondo de muchas discusiones filosóficas en la primera mitad del siglo XX es la identificación de causalidad con determinismo [...]. Esta identificación tiene profundas implicaciones para la manera como se entiende la relación entre causalidad e historia” (Martínez 1998, p. 203). Sin embargo, este ideal se ve mermado ante la posibilidad de explicar científicamente también a partir de causas no deterministas que admiten contingencia e historia, tal como es implicado por la evolución. 
variacional y estadística, más acorde con la naturaleza del mundo viviente que la del enfoque tipológico más tradicional (platónico/esencialista). De acuerdo a esta idea, las explicaciones biológicas predarwinianas, de corte tipológico esencialista, serían inadecuadas para explicar los hechos naturales y que le interesan a la biología evolutiva: la variación y sus frecuencias de ocurrencia en una población. Por esta razón, con Darwin se inicia una nueva manera de hacer investigación, orientada a responder un novedoso conjunto de preguntas más acordes con la realidad biológica, preguntas que estaban ausentes antes de la formulación de la teoría de la evolución por selección natural. En pocas palabras, "Darwin reemplazó el pensamiento tipológico por el pensamiento poblacional" (Mayr [1975] 2006, p. 326). Es decir, para Mayr el pensamiento poblacional es una doctrina metafísica que se opone al pensamiento tipológico o esencialismo Ahora bien, ¿sobre qué bases está construida la dicotomía? Veamos los siguientes pasajes de Mayr, usualmente citados en la literatura que trata este tema:

It was Darwin's genius to see that this uniqueness of each individual is not limited to the human species but is equally true for every sexually reproducing species of animal and plant. Indeed, the discovery of the importance of the individual became the cornerstone of Darwin's theory of natural selection. It eventually resulted in the replacement of essentialism by population thinking, which emphasized the uniqueness of the individual and the critical role of individuality in evolution [...] And variation, which had been irrelevant and accidental for the essentialist, now became on of the crucial phenomena of living nature (Mayr 1991, p. 42).

Typological thinking no doubt had its roots in the earliest efforts of primitive man to classify the bewildering diversity of nature into categories. The eidos of Plato is the formal codification of this form of thinking [...] Since there is no gradation between types, gradual evolution is a logical impossibility for the typologist. Evolution, if it occurs, has to proceed in steps or jumps. The assumptions of population thinking are diametrically opposed to those of the typologist. The populationist stresses the uniqueness of everything in the organic world. What is true for the human species - that no two individuals are alike- is equally true for all other species of animals and plants. Indeed, even the same individual changes continuously throughout its lifetime and when placed into different environments. All organisms and organic phenomena are composed of unique features and can be described collectively only in statistical terms. Individuals, or any kind of organic entities, form populations of which we can determine the arithmetic mean and the statistics of variation. Averages are merely statistical abstractions, only the individuals of which populations are made have reality. The ultimate conclusions of the population thinker and of the typologist are precisely the opposite. For the typologist the type (eidos) is real and the variation an illusion, while for the populationist the type (average) is an abstraction and only the variation is real. No two ways of looking at nature could be more different (Mayr [1975] 2006, pp. 326-27).

Los pasajes citados reflejan de forma concisa las concepciones que están detrás de la división entre ‘tipo' y 'población'. De allí podríamos extraer que:

(a) el gran logro de Darwin (asociado al descubrimiento de la selección natural) fue reemplazar el pensamiento esencialista por el pensamiento poblacional;

(b) hay una relación directa (de identidad) entre tipo y eidos platónico (arquetipos universales, eternos e inmutables);

(c) tanto el enfoque poblacional como el tipológico tienen un componente ontológico: se concentran en la determinación de la realidad de las entidades que habitan el mundo. Para el pensador poblacional darwiniano los tipos no son reales, sólo los individuos disímiles y las poblaciones que éstos componen (no hay dos individuos idénticos en el mundo). Por el contrario, para el tipologista los tipos son reales, la variación no.

De lo anterior se puede concluir que una biología sustentada en un enfoque tipológico es errada, pues fundamenta sus bases teóricas y su metodología empírica en entidades inexistentes: los tipos universales. Por el contrario, la biología en general (y no sólo la biología evolutiva) debe hacer una 
aproximación poblacional, pues es allí en donde se atiende la individualidad y su complejidad. De acuerdo con Mayr, es por esta razón que gracias a Darwin se supera el erróneo 'pensamiento tipológico', para dar paso al 'pensamiento poblacional'

Por su parte, siguiendo en lo fundamental a Mayr (aunque con ciertas divergencias), Sober reformula la distinción tipo/población, ligando el primero (el pensamiento tipológico) a la tradición aristotélica y su modelo de 'estado natural' y fuerzas de interferencia, mientras que el segundo (el pensamiento poblacional) es una aplicación del pensamiento estadístico y de probabilidades (Sober [1980] 2006). Dice Sober:

The essentialist hoped to penetrate the veil of variability found within species by discovering some natural tendency which each individual in the species possesses. This natural tendency was to be a dispositional property which would be manifest, were interfering forces not at work. Heterogeneity is thus the result of a departure from natural state. But, with the development of evolutionary theory, it turned out that no such property was available to the essentialist's causal hypothesis about the origins of variability [...]. At the same time that evolutionary theory undermined the essentialist's model of variability, it also removed the need for discovering species essences. Characteristics of populations do not have to be defined in terms of characteristics of organisms for population concept to be coherent and fruitful. Population biology attempts to formulate generalizations about kinds of populations. [...] Essentialism lost its grip, when populations came to be thought of as real (Sober [1980] 2006, pp. 353. 354).

La similitud entre lo que exponen Sober y Mayr es evidente, sobre todo en la adjudicación de realidad a las poblaciones y la negación de ésta a los tipos esenciales que, se supone, hace la teoría darwiniana. Pero la formulación directa de la relación de identidad entre pensamiento poblacional y análisis estadístico es hecha por Sober en su influyente libro The Nature of Selection ([1984] 1993). Examinando cuáles son los alcances de la revolución darwiniana, para Sober lo novedoso de la teoría de la evolución y su proceso de selección natural es su carácter estrictamente poblacional: la selección natural explicaría la persistencia, difusión y porcentaje particular de los individuos y sus rasgos en una población (Sober [1984] 1993). Este es el carácter estadístico de la evolución. ${ }^{18}$

Podríamos resumir la posición de Mayr y Sober diciendo que, para ellos, el gran logro de Darwin es reemplazar el pensamiento tipológico de la biología, el cual trata de esencias inexistentes; imponiendo un marco conceptual más acertado para entender la realidad biológica, consistente en poblaciones compuestas de individuos no similares (diversidad). Por esta razón, el pensamiento poblacional, la nueva forma de entender la naturaleza debe ser estadístico: son los porcentajes de distribución de las variantes de individuos y rasgos en la población lo que estudia la evolución. Son los cambios en las frecuencias de genes y rasgos en una población lo que determina la evolución, cambios causados principalmente por la selección natural (y en una menor medida, por otros procesos tales como deriva génica, migración o eventos estocásticos). Un enfoque tipológico, en un mundo tan variable y en permanente cambio, no puede más que ser equivocado. En palabras de Wuketits: “Darwin’s theory [...] meant no less than a shift from a static (essentialist) to dynamic thinking. This might be regarded as

\footnotetext{
${ }^{18}$ Vale la pena señalar la coincidencia con Peirce al respecto: "The Darwinian controversy is, in large part, a question of logic. Mr. Darwin proposed to apply the statistical method to biology. The same thing has been done in a widely different branch of science, the theory of gases. Though unable to say what the movements of any particular molecule of gas would be on a certain hypothesis regarding the constitution of this class of bodies, Clausius and Maxwell were yet able, eight years before the publication of Darwin's immortal work, by the application of the doctrine of probabilities, to predict that in the long run such and such a proportion of the molecules would, under given circumstances, acquire such and such velocities; that there would take place, every second, such and such a relative number of collisions, etc.; and from these propositions were able to deduce certain properties of gases, especially in regard to their heat-relations. In like manner, Darwin, while unable to say what the operation of variation and natural selection in any individual case will be, demonstrates that in the long run they will, or would, adapt animals to their circumstances. Whether or not existing animal forms are due to such action, or what position the theory ought to take, forms the subject of a discussion in which questions of fact and questions of logic are curiously interlaced" (Peirce, 1877, pp. 2-3).
} 
the very meaning of what has been frequently called 'Darwinian Revolution', for Darwin showed that what is real in nature is not the type, but variation" $(2005$, p. 57).

\subsection{Algunas críticas}

Varias críticas han surgido al respecto de esta dicotomía, creo que de manera justificada. En otro lugar he argumentado (Martínez \& Andrade 2011) que Darwin y la teoría evolutiva no rechaza la idea de tipo, sólo se reformula su concepto en términos de esencias históricas. Esto implicaría que desde la biología evolutiva no se refute al esencialismo, sino que se re-conceptualiza a partir de un análisis factual (se rechaza el esencialismo platónico para adoptar un esencialismo histórico). Recordemos que Darwin mismo enfatiza que la unidad de tipo se explica por ancestro común (Martínez \& Andrade 2011; véase también Griffths 1999, Okasha 2002, LaPorte 2004).

Otra crítica es la señalada por Ariew (2008), quien argumenta que el pensamiento poblacional es ante todo un cambio metodológico, no de metafísica. Para Ariew, "There must be more to Darwin's great insight than the rather silly metaphysics that Mayr attributes to essentialists and Darwinians." (2008, p. 65) Según Ariew, consciente de la existencia tanto de regularidad (macro) como de variación (micro) en la naturaleza, el aporte de Darwin es conectarlas a las dos: propone que la evolución es una regularidad a gran escala que emerge de las vidas variadas de los individuos a menor escala.

\section{Conclusiones}

A lo largo de estas páginas he expuesto algunas de las importantes consecuencias que la teoría de la evolución por selección natural ha tenido en la filosofía. La lista tratada está lejos de cubrir todas las temáticas y detalles, pero nos da una buena idea de la relevancia que los argumentos evolucionistas han tenido y continúan teniendo en varios de los temas filosóficos centrales. Muchos conceptos, problemas, enfoques y metodologías de la filosofía han sido influidos tanto por la creciente evidencia empírica como por el trabajo teórico cada vez más profundo en teoría evolutiva. Confiadamente podríamos decir que la filosofía contemporánea, de una manera muy saludable, no ha sido ajena a una de nuestras mejores teorías científicas. Más aún, el proyecto naturalista ha logrado consolidarse, convirtiéndose en uno de los programas de investigación filosófica más extendidos: hoy en día oímos hablar frecuentemente de metafísica, epistemología, ética o mentes naturalizadas. Claro (y no menos importante), afortunadamente no han faltado los espíritus críticos que, desde la misma filosofía, han puesto en duda el pretendido éxito de la teoría de la evolución para iluminar debates y proveer de soluciones definitivas o parciales a los problemas filosóficos milenarios. Digo afortunadamente, pues la discusión entre filosofía y biología es una que debe darse permanentemente y de manera profunda, procurando siempre una rica y fructífera retroalimentación.

\section{Bibliografía}

Abrantes, P. (2011), "Introdução: o que é filosofia da biologia", en Abrantes, P. (ed.), Filosofia da biologia, São Paulo: Artmed.

Ariew, A. (2008), "Population Thinking", en Ruse, M. (ed.), The Oxford Handbook of Philosophy of Biology, New York: Oxford University Press, pp. 64-86.

Axelrod, R. y W. Hamilton (1981), “The Evolution of Cooperation”, Science, New Series 211(4489): 1390-1396. 
Barahona, A. y S. Martínez (1998), "Teleología y biología”, en Martínez, S. y A. Barahona (eds.), Historia y explicación en biologia, México: FCE-UNAM, pp. 419-430.

Beer, G. (1983), Darwin's Plots: Evolutionary Narrative in Darwin, George Eliot, and Nineteenth-Century Fiction, London: Routledge \& Kegan Paul.

Bell, C. (1834), The Bridgewater Treatises on the Power, Wisdom, and Goodness of God, As Manifested in the Creation.

Birch, J. y S. Okasha (2015), “Kin Selection and its Critics”, Bioscience 65(1): 22-32.

Borrello, M. (2012), Evolutionary Restraints: The Contentious History of Group Selection, Chicago: The University of Chicago Press.

Bradie, M. (1986), “Assessing Evolutionary Epistemology”, Biology $\mathcal{E}$ Philosophy 1(4): 401-459.

Bradie, M. (2012), "Evolutionary Epistemology", The Stanford Encyclopedia of Philosophy (Winter 2012 edition). Accesible en: http://plato.stanford.edu/archives/win2012/entries/epistemology-evolutionary/.

Campbell, D.T. (1974), "Evolutionary Epistemology", en Schilpp, P.A. (ed.), The Philosophy of Karl R. Popper, LaSalle: Open Court, pp. 412-463.

Caponi, G. (2011), La segunda agenda darwiniana, México: CEFPSVLT.

Cosmides, L. y J. Tooby (1992), "Cognitive Adaptations for Social Exchange”, en Barkow, J., Cosmides, L. y J. Tooby (eds.), The Adapted Mind: Evolutionary Psychology and the Generation of Culture, New York: Oxford UP, pp. 163-228.

Caponi, G. (2012), Função e desenho na biologia contemporânea, São Paulo: Editora 34-Associação Scientiae Studia.

Darwin, C. (1859), On the Origin of Species by Means of Natural Selection, London: John Murray.

Darwin, C. (1871), The Descent of Man, and Selection in Relation to Sex, Cambridge: D. Appleton and Company.

Darwin, C. (1972), The Expression of the Emotions in Man and Animals, London: John Murray.

Diéguez, A. (2012), La vida bajo escrutinio, España: Biblioteca Buridán.

Fodor, J. (1983), The Modularity of Mind: An Essay on Faculty Psychology, Cambridge: MIT Press.

Ginsborg, H. (2006), "Kant's Biological Teleology and its Philosophical Significance”, en Bird, G. (ed.), The Blackwell Companion to Kant, Oxford: Blackwell Publishing, pp. 455-469.

Gould, S.J. (2002), The Structure of Evolutionary Theory, Cambridge: Belknap Press.

Greene, J. (2007), “The Secret Joke of Kant's Soul”, en Sinnott-Armstrong, W. (ed.), Moral Psychology, Vol. 3: The Neuroscience of Morality: Emotion, Disease, and Development, Cambridge: MIT Press, pp. 35-117.

Greene, J. (2015), “The Rise of Moral Cognition”, Cognition 135: 39-42.

Griffiths, P. (1999), "Squaring the Circle: Natural Kinds with Historical Essences", en Wilson, R.A. (ed.), Species: New Interdisciplinary Essays, Cambridge: MIT Press, pp. 209-228.

Guillaumin, G. (2009), Raices metodológicas de la teoría de la evolución de Charles Darwin, Barcelona-México: AnthroposUAM-I.

Haidt, J. (2001), "The Emotional Dog and Its Rational Tail: A Social Intuitionist Approach to Moral Judgment", Psychological Review 108(4): 814-834.

Hauser, M. (2006), Moral Minds, New York: Harper Collins.

Hösle, V. y C. Illies (2005), Darwinism $\mathcal{E}$ Philosophy, University of Notre Dame Press.

Hull, D. (1969), "What Philosophy of Biology is Not", Journal of the History of Biology 2: 241-68.

Hull, D. (1988), Science as a Process: An Evolutionary Account of the Social and Conceptual Development of Science, Chicago: The University of Chicago Press.

Hull, D. (2008), "The History of the Philosophy of Biology", The Oxford Handbook of Philosophy of Biology, New York: Oxford UP, pp. 11-33. 
Joyce, R. (2006), The Evolution of Morality, Cambridge: MIT Press.

Kitcher, P. (2006), "Between Fragile Altruism and Morality: Evolution and the Emergence of Normative Guidance", en Boniolo, G. y G. De Anna (eds.), Evolutionary Ethics and Contemporary Biology, Cambridge: Cambridge University Press, pp. 159-77.

Ladyman, J. y D. Ross (2010), Every Thing Must Go, Oxford: Oxford University Press.

Laland, K., Sterelny, K., Odling-Smee, J., Hoppitt, W. y T. Uller (2011), "Cause and Effect in Biology Revisited: Is Mayr's Proximate-Ultimate Dichotomy Still Useful?”, Science (334): 1512-1516.

LaPorte, J. (2004), Natural Kinds and Conceptual Change, Cambridge: Cambridge University Press.

Lewens, T. (2008), "The Origin and Philosophy", en Richards, R. y M. Ruse (eds.), The Cambridge Companion to the Origins of Species, Cambridge: Cambridge University Press, pp. 314-332.

Loeb, J. (1912), The Mechanistic Conception of Life, Chicago: The University of Chicago Press.

Lorenz, K. (1977), Behind the Mirror, London: Methuen.

Martínez, M. (2003), "La falacia naturalista y el argumento de la pregunta abierta”, Universitas Philosophica 40(1): 65-88.

Martínez, M. y A. Moya (2011), “Natural Selection and Multilevel Causation”, Philosophy Eु Theory in Biology (3)e: 302.

Martínez, M. y E. Andrade (2011), “A contingência dos padrões de organização biológica: superando a dicotomia entre pensamento tipológico e populacional”, en Abrantes, P. (ed.), Filosofia da biologia, Porto Alegre: ARTMED, pp. $145-161$.

Martínez, M. y M. Esposito (2014), “Multilevel Causation and the Extended Synthesis”, Biological Theory 9(2): $209-220$.

Martínez, S. (1998), “Sobre la relación entre historia y causalidad en biología”, en Martínez, S. y A. Barahona (eds.), Historia y explicación en biología, México: FCE-UNAM, pp. 23-41.

Martínez-Contreras, J. (2011), "How Apes Became Human”, en Martínez-Contreras, J. y A. Ponce de León (eds.), México: Siglo XXI-UV, pp. 396-409.

Mayr, E. (1961), “Cause and Effect in Biology”, Science 134(3489): 1501-1506.

Mayr, E. (1991), One Long Argument, Cambridge: Harvard University Press.

Mayr, E. (1994), “Reply to John Beatty”, Biology Eु Philosophy 9: 357-358.

Mayr, E. (2002), What Evolution Is, New York: Basic Books.

Mayr, E. ([1975] 2006), “Typological versus Population Thinking”, en Sober, E. (ed.), Conceptual Issues in Evolutionary Biology, Cambridge, MA: MIT Press, pp. 325-328.

Mithen, S. (1998), The Prehistory of the Mind: A Search for the Origins of Art, Religion, and Science, London: Thames and Hudson.

Moore, G. E. ([1903] 1997), Principia Ethica, México: UNAM.

Nagel, E. (1961), The Structure of Science: Problems in the Logic of Scientific Explanation, New York: Harcourt, Brace and World.

Nagel, E. (1951), "Mechanistic Explanation and Organismic Biology", Philosophy and Phenomenological Research 11: $327-$ 338.

Oppenheim, P. y H. Putnam (1958), "Unity of Science as a Working Hypothesis”, en Feigl, H., Scriven, M. y G. Maxwell (eds.), Minnesota Studies in the Philosophy of Science, Vol. 2, Minneapolis: University of Minnesota Press, pp. 3-36.

Okasha, S. (2002), “Darwinian Metaphysics: Species and The Question Of Essentialism”, Synthese 131(2): 191-213.

Paley, W. ([1802] 1836), Natural Theology, Londres: Charles Knight.

Peirce, C.S. (1877), "The Fixation of Belief”, Popular Science Monthly 12: 1-15. 
Pinker, S. (1997), How the Mind Works, New York: Norton.

Popper, K. (1992), Objective Knowledge: An Evolutionary Approach, Oxford: Clarendon Press.

Rosas, A. (2000), "Explicación y justificación: hacia el naturalismo en la filosofía moral”, en Botero, J.J., Ramos, J. y A. Rosas (eds.), Mentes reales: la ciencia cognitiva y la naturalización de la mente, Bogotá: Siglo de Hombres editores, pp. 101-118.

Rosas, A. (2004), “La evolución de la cooperación”, en Hoyos, L.E. (ed.), Lecciones de Filosofía Política, Bogotá: Universidad Nacional, pp. 347-371.

Rosenberg, A. (2006), Darwinian Reductionism: Or, How to Stop Worrying and Love Molecular Biology, Chicago: Chicago University Press.

Ruse, M. (1986), Taking Darwin Seriously, New York: Prometheus.

Ruse, M. (1980), “Darwin and Group Selection”, Annals of Science 37: 615-630.

Ruse, M. (2009), Philosophy after Darwin: Classic and Contemporary Readings, Princeton: Princeton University Press.

Sober, E. ([1983] 2003), The Nature of Selection: Evolutionary Theory in Philosophical Focus, Chicago: The University of Chicago Press.

Sober, E. ([1975] 2006), “Evolution, Population Thinking and Essentialism”, en Sober, E. (ed.), Conceptual Issues in Evolutionary Biology, Cambridge: MIT Press, pp. 329-362.

Sober, E. (2011), Did Darwin Write the Origin Backwards?, New York: Prometheus.

Sober, E. y D.S Wilson (1998), Unto Others: The Evolution and Psychology of Unselfish Behavior, Cambridge: Hardvard University Press

Street, S.A. (2006), "Darwinian Dilemma for Realist Theories of Value”, Philosophical Studies 127: 109-166.

Tooby, J. y L. Cosmides (2005), “Conceptual Foundations of Evolutionary Psychology”, en Buss, D. (ed.), The Handbook of Evolutionary Psychology, Hoboken: John Wiley \& Sons, Ltd., pp. 5-67.

Toulmin, S. (1972), Human Understanding: The Collective Use and Evolution of Concepts, Princeton: Princeton University Press.

Trivers, R. (1971), “The Evolution of Reciprocal Altruism”, Quarterly Review of Biology 46: 35-57.

Walsh, D. (2008), “Teleology”, en Ruse, M. (ed.), The Oxford Handbook of Philosophy of Biology, New York: Oxford University Press, pp. 113-137.

Williams, G.C. (1966), Adaptation and Natural Selection, Princeton: Princeton University Press.

Wilson, D.S. y E.O. Wilson (2008), “Evolution for the Good of the Group”, American Scientist 96(5): 380-389.

Wilson, E.O. (1975), Sociobiology: The New Synthesis, Cambridge: Harvard University Press.

Wilson, E.O. (1978), On Human Nature, Cambridge: Harvard University Press.

Wuketits, F. (2005), “The Theory of Biological Evolution: Historical and Philosophical Aspects”, en Ayala, F.J. y F.M. Wuketits (eds.), Handbook of Evolution, Weinheim: Wiley, pp. 57-86.

Wynne, E. (1962), Animal Dispersion in Relation to Social Behavior, Londres: Oliver \& Boyd. 